\title{
Clinical and virological course of patients with coronavirus disease 2019 in Jiangsu province, China: a retrospective, multi-center cohort study
}

\author{
Rui Huang ${ }^{1 \dagger}$, Li Zhu ${ }^{2 \dagger}$, Leyang Xue ${ }^{3 \dagger}$, Xuebing Yan ${ }^{4 \dagger}$, Jian Wang ${ }^{1 \dagger}$, Songping Huang ${ }^{5 \dagger}$, Biao Zhang ${ }^{6}$, \\ Tianmin $\mathrm{Xu}^{7}$, Fang $\mathrm{Ji}^{4}$, Chunyang $\mathrm{Li}^{4}$, Fang Ming ${ }^{5}$, Yun Zhao ${ }^{8}$, Yang $\mathrm{Li}^{9}$, Juan Cheng ${ }^{10}$, Yinling Wang ${ }^{2}$, \\ Huaping Shao ${ }^{11}$, Shuqin Hong ${ }^{12}$, Kang Chen ${ }^{13}$, Xiang-an Zhao ${ }^{14}$, Dawen Sang ${ }^{10}$, Lei Zou ${ }^{10}$, Haiyan Zhao ${ }^{11}$, \\ Xinying Guan ${ }^{15}$, Xiaobing Chen ${ }^{16}$, Biyun Xu ${ }^{17}$, Juan Xia ${ }^{1}$, Yuxin Chen ${ }^{18}$, Xiaomin Yan ${ }^{1}$, Jie Wei ${ }^{19}$, Jiacheng Liü ${ }^{20}$, \\ Longgen $\mathrm{Liu}^{7^{*}}$, Chuanwu Zhu² ${ }^{2^{*}}$ and Chao $\mathrm{Wu}^{1 *}$
}

\begin{abstract}
Background: The clinical and virological course of patients with coronavirus disease 2019 (COVID-19) are lacking. We aimed to describe the clinical and virological characteristics of COVID-19 patients from 10 designated hospitals in 10 cities of Jiangsu province, China. The factors associated with the clearance of SARS-CoV-2 were investigated.

Methods: A total of 328 hospitalized patients with COVID-19 were retrospectively recruited. The epidemiological, clinical, laboratory, radiology and treatment data were collected. The associated factors of SARS-CoV-2 clearance were analyzed.

Results: The median duration of hospitalization was 16.0 days (interquartile range [IQR] 13.0-21.0 days). On multivariate Cox regression analysis, age $>60$ years (hazard ratio [HR] 0.643, 95\% confidence interval [CI] 0.454-0.911, $P=0.013$ ) was associated with the delayed SARS-CoV-2 clearance, while the atomized inhalation of interferon $\mathrm{a}-2 \mathrm{~b}$ could improve the clearance of SARS-CoV-2 (HR, 1.357, 95\% Cl 1.050-1.755, $P=0.020)$. Twenty-six (7.9\%) patients developed respiratory failure and 4 (1.2\%) patients developed ARDS. Twenty (6.1\%) patients were admitted to the ICU, while no patient was deceased.
\end{abstract}

Conclusions: Our study found that age $>60$ years was associated with the delayed SARS-CoV-2 clearance, while treated with atomized inhalation of interferon $a-2 b$ could promote the clearance of SARS-CoV-2.

Keywords: Coronavirus disease 2019, SARS-CoV-2, Viral, Clearance

\footnotetext{
*Correspondence: ssewllg@163.com; zhuchw@126.com; dr.wu@nju.edu.cn ${ }^{\dagger}$ Rui Huang, Li Zhu, Leyang Xue, Xuebing Yan, Jian Wang, and Songping Huang have contributed equally to this work

${ }^{1}$ Department of Infectious Diseases, Nanjing Drum Tower Hospital, The Affiliated Hospital of Nanjing University Medical School, Nanjing, China 2 Department of Infectious Diseases, The Affiliated Infectious Diseases Hospital of Soochow University, Suzhou, China

${ }^{7}$ Department of Infectious Diseases, The Third People's Hospital of Changzhou, Changzhou, China

Full list of author information is available at the end of the article
}

\section{Background}

The outbreak of coronavirus disease 2019 (COVID-19) in Wuhan, China, caused by the severe acute respiratory syndrome coronavirus 2 (SARS-CoV-2) [1], continues to spread among humans. As of May 14, 2021, $160,686,749$ confirmed cases and 3,335,948 deaths have been reported [2]. The clinical spectrum of SARS-CoV-2 infection presents from asymptomatic infection to severe 
pneumonia with fatal outcomes [3]. Although the epidemiological, radiologic and clinical characteristics of COVID-19 patients have been reported in several studies [4-6], the details of clinical and virological course of patients with COVID-19 are lacking. Zhou et al. described the clinical course and clearance of SARSCoV-2 during hospitalization of COVID-19 patients with a definite clinical outcome in Wuhan [7]. However, several studies have demonstrated that the characteristics of patients outside of Wuhan were different from those initially reported patients in Wuhan [8-10]. Furthermore, the factors which affect the clearance of SARS-CoV-2 have not been identified.

In this multi-center study, we described the clinical and virological characteristics of patients with COVID-19 and explored the factors associated with the clearance of SARS-CoV-2 in Jiangsu province, China.

\section{Methods}

\section{Patients}

Between January 18, 2020 and February 29, 2020, patients confirmed with COVID-19 were retrospectively recruited from ten designated hospitals in 10 cities of Jiangsu province, China. The last follow-up date was March 10, 2020.

COVID-19 was confirmed by quantitative real-time reverse transcription polymerase chain reaction (RTPCR) test using the same protocol described previously [11]. Throat swab specimens of all confirmed patients was collected on admission and during hospitalization. Laboratory tests were carried out in these designated hospitals and local Center for Disease Control and Prevention.

\section{Procedures}

We collected and reviewed all the medical records of confirmed COVID-19 patients in each medical center. Data of patients regarding the epidemiological, clinical, laboratory, radiology and treatment were collected. The criteria of acute respiratory distress syndrome (ARDS) was based on the corresponding guidelines [12]. The management strategies and discharge criteria of COVID-19 in different hospitals were in accordance with the guidelines by the Chinese National Health Commission (Trial Version 5) [13]. The frequency of RT-PCR testing was determined by specific clinicians who were responsible for the management of patients according to the disease progression. The time of SARS-CoV-2 RNA negative was defined as two consecutively negative SARS-CoV-2 nucleic acid by RT-PCR test separated by at least 1 day. The days of viral clearance were calculated by the time of SARS-CoV-2 RNA negative minus the time of initial symptoms.
All patients were confirmed by throat swab samples tested by a RT-PCR in accordance with the protocol by the World Health Organization [14].

\section{Statistical analysis}

The median and interquartile range (IQR) were used to present the continuous variables. Categorical variables were described as the counts and percentages. The independent group $t$ tests (normal distribution) and Mann-Whitney $U$ (non-normal distribution) were used to compare continuous variables between groups. Chisquare or Fisher's exact test was used to compare the categorical variables. Cox regression was used to analyze the associated factors of SARS-CoV-2 clearance. Variables having $P$ values $<0.1$ in the univariate analysis were further used for a multivariate Cox regression analysis. Accumulative clearance of SARS-CoV-2 were generated using Kaplan-Meier method. Log-rank test was used to test the equivalences of the survival curves. $P$ values for multiple comparisons was not corrected in our study. $P<0.05$ was considered to be statistically significant. SPSS version 22.0 software (SPSS Inc., Chicago, IL, United States) was used for the data analysis.

\section{Results}

Demographic and epidemiologic characteristics

A total of 342 hospitalized patients during January 18, 2020 to February 29, 2020 who were confirmed as SARS-CoV-2 infection were identified. Fourteen asymptomatic patients were also excluded. Finally, 328 COVID-19 patients were included for the analysis (Fig. 1). All patients were discharged before March 10, 2020. The median age of the patients was 45.0 (IQR $33.0-55.0)$ years (Table 1). Most (84.8\%) of the patients were aged $<60$ years. $176(53.7 \%)$ patients were male. The median body mass index (BMI) was 24.2 (IQR 22.2-26.2) $\mathrm{kg} / \mathrm{m}^{2} .88(26.8 \%)$ patients had at least one underlying comorbidity, including hypertension (50 [15.2\%]), type 2 diabetes (27 [8.2\%]), chronic lung diseases (12 [3.7\%]), chronic liver diseases (9 $[2.7 \%])$, cardiovascular diseases (6 [1.8\%]), and malignant tumors (5 [1.5\%]). The median time from symptom onset to admission was 5.0 (IQR 2.0-8.0 days) days. The median duration of hospitalization was 16.0 (IQR 13.0-21.0) days. All patients cleared the SARS-CoV-2 during the study period and the median duration of viral clearance after COVID-19 onset was 17.0 (IQR 13.0-21.0) days. The distributions of viral clearance days among these patients were presented in Fig. 2.

\section{Clinical features and laboratory abnormalities}

Fever (237 [72.3\%]) and cough (200 [61.0\%]) were the most common symptoms (Table 2). 85 (25.9\%) patients 
342 confirmed COVID-19 patients between January 18, 2020 and February 29, 2020

excluded

Asymptomatic patients $(n=14)$

328 COVID-19 patients were enrolled

Fig. 1 Flow diagram describing the selection of study population

Table 1 Demographic and epidemiologic characteristics of patients with coronavirus disease 2019

\begin{tabular}{lc}
\hline Variables $(\mathbf{n}[\%]$ or median [IQR]) & All patients $(\mathbf{n}=\mathbf{3 2 8})$ \\
\hline Age (year) & $45.0(33.0,55.0)$ \\
Age range & $278(84.8)$ \\
$<60$ & $50(15.2)$ \\
$\geq 60$ & \\
Gender & $176(53.7)$ \\
Male & $152(46.3)$ \\
Female & $24.2(22.2,26.2)$ \\
BMl (kg/m²)* & \\
Comorbidities & $88(26.8)$ \\
Any comorbidity & $50(15.2)$ \\
Hypertension & $27(8.2)$ \\
Type 2 diabetes & $12(3.7)$ \\
Chronic lung diseases & $9(2.7)$ \\
Chronic liver diseases & $6(1.8)$ \\
Cardiovascular diseases & $5(1.5)$ \\
Malignant tumors & $23(7.0)$ \\
Smoking history &
\end{tabular}

$P C R$ polymerase chain reaction, IQR interquartile range, $B M I$ body mass index *Available for 292 patients

showed leukopenia and 99 (30.2\%) patients had lymphopenia on admission. Chest CT scans were performed at the time of admission, $93.3 \%$ of the patients had abnormal results. The most common patterns on chest CT

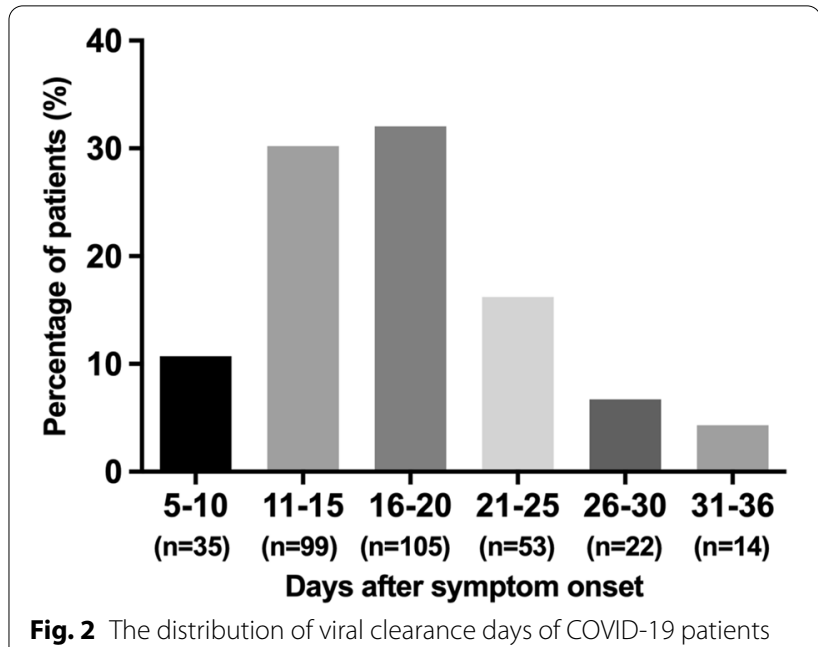

images were ground-glass opacity (73.2\%). No CT abnormality was found in $22(6.7 \%)$ patients.

\section{Complications, treatment and outcomes}

Oxygen therapy was administered in 203 (61.9\%) of patients and mechanical ventilation in $15(4.6 \%)$ of patients. Majority of the patients $(78.0 \%)$ received empirical antibiotic therapy. Glucocorticoids were given to 84 (25.6\%) patients. Most of the patients received at least one antiviral drug (atomized inhalation of interferon $\alpha-2 b, 55.2 \%$; lopinavir/ritonavir, $74.7 \%$; and arbidol, 
Table 2 Clinical Characteristics and laboratory findings of patients with coronavirus disease 2019

\begin{tabular}{|c|c|}
\hline Variables ( $\mathrm{n}[\%]$ or median [IQR]) & All patients $(n=328)$ \\
\hline \multicolumn{2}{|l|}{ Onset signs and symptoms } \\
\hline Fever & $237(72.3)$ \\
\hline Cough & $200(61.0)$ \\
\hline Fatigue & $69(21.0)$ \\
\hline Sore throat & $34(10.4)$ \\
\hline Muscle ache & $35(10.7)$ \\
\hline Shortness of breath & $27(8.2)$ \\
\hline Headache & $20(6.1)$ \\
\hline \multicolumn{2}{|l|}{ Laboratory findings } \\
\hline WBC $\left(\times 10^{9} / L\right)^{*}$ & $4.8(3.8,6.1)$ \\
\hline Decreased & $85(25.9)$ \\
\hline Lymphocyte $\left(\times 10^{9} / \mathrm{L}\right)^{\#}$ & $1.2(0.9,1.6)$ \\
\hline Decreased & $99(30.2)$ \\
\hline $\mathrm{Hb}(\mathrm{g} / \mathrm{L})$ & $138.0(128.0,151.0)$ \\
\hline $\operatorname{PLT}\left(\times 10^{9} / \mathrm{L}\right)$ & $174.5(140.0,219.3)$ \\
\hline $\mathrm{ALT}(\mathrm{U} / \mathrm{L})$ & $25.0(19.0,35.3)$ \\
\hline $\mathrm{LDH}(\mathrm{U} / \mathrm{L})$ & $243.0(179.3,390.3)$ \\
\hline $\mathrm{ALB}(\mathrm{g} / \mathrm{L})$ & $40.1(37.1,43.3)$ \\
\hline $\mathrm{Cr}(\mu \mathrm{mol} / \mathrm{L})$ & $64.0(52.0,78.0)$ \\
\hline $\mathrm{PT}(\mathrm{s})$ & $12.8(12.0,13.4)$ \\
\hline D-Dimer (mg/L) & $0.2(0.1,0.4)$ \\
\hline \multicolumn{2}{|l|}{ Chest CT findings } \\
\hline No pneumonia & $22(6.7)$ \\
\hline Unilateral pneumonia & $49(14.9)$ \\
\hline Bilateral pneumonia & $257(78.4)$ \\
\hline Ground glass opacity & $240(73.2)$ \\
\hline Time from symptom onset to admission (days) & $5.0(2.0,8.0)$ \\
\hline
\end{tabular}

$P C R$ polymerase chain reaction, $I Q R$ interquartile range, $W B C$ white blood cells, $H b$ hemoglobin, $P L T$ platelet, $T B$ total bilirubin, $A L T$ alanine transaminase, $L D H$ lactate dehydrogenase, $A L B$ albumin, $\mathrm{Cr}$ creatinine, $P T$ prothrombin time *The normal ranges of WBC were $4-10 \times 10^{9} / \mathrm{L}$ or $3.5-9.5 \times 10^{9} / \mathrm{L}$ in different hospitals and decreased means below the lower limit of the normal range

\# The normal ranges of lymphocyte were $0.8-4 \times 10^{9} / \mathrm{L}$ or $1.1-3.2 \times 10^{9} / \mathrm{L}$ in different hospitals and decreased means below the lower limit of the normal range

43.9\%). The complications included respiratory failure (26 [7.9\%] patients), and acute respiratory distress syndrome (4 [1.2\%] patients). 20 (6.1\%) patients were admitted to the ICU. However, no patient died in our study (Table 3) [15].

\section{Associated factors of SARS-CoV-2 clearance}

The associated factors of SARS-CoV-2 clearance in COVID-19 patients were analyzed by cox regression analysis (Table 4). The univariate analysis showed that the factors for patients with delayed clearance of SARS$\mathrm{CoV}-2$ were age $>60$ years (hazard ratio [HR], 0.610, $95 \%$ confidence interval $[\mathrm{CI}](0.450,0.826), P=0.001)$,
Table 3 Treatment and outcomes of patients with coronavirus disease 2019

\begin{tabular}{ll}
\hline Variables (n [\%]) & All patients $(\mathbf{n}=\mathbf{3 2 8})$ \\
\hline Oxygen therapy & $203(61.9)$ \\
Mechanical ventilation & $15(4.6)$ \\
Drug treatment & \\
Atomized inhalation of interferon a-2b & $181(55.2)$ \\
Lopinavir/ritonavir & $245(74.7)$ \\
Arbidol & $144(43.9)$ \\
Antibiotic & $256(78.0)$ \\
Glucocorticoid & $84(25.6)$ \\
Gamma globulin & $44(13.4)$ \\
Complications & \\
Respiratory failure & $26(7.9)$ \\
ARDS & $4(1.2)$ \\
Outcome & \\
Severe illness & $35(10.7)$ \\
Admission to ICU & $20(6.1)$ \\
Death & 0
\end{tabular}

$P C R$ polymerase chain reaction, $A R D S$ acute respiratory distress syndrome, $I C U$ intensive care unit

while the atomized inhalation of interferon $\alpha-2 b(H R$, $1.355,95 \%$ CI $1.088,1.688, P=0.007)$ could improve the clearance of SARS-CoV-2. On the multivariate analysis, atomized inhalation of interferon $\alpha-2 b$ (HR, 1.357, 95\% CI 1.050-1.755, $P=0.020$ ) was associated with an increased improvement of SARS-CoV-2 clearance compared with patients not receiving atomized inhalation of interferon $\alpha-2 b$, while age $>60$ years $(\mathrm{HR}, 0.643$, 95\% CI $0.454-0.911, P=0.013)$ was associated with a prolonged time to viral clearance compared with patients $\leq 60$ years by adjusting sex, BMI, LDH, hypertension, and diabetes.

Accumulative clearance of SARS-CoV-2 was presented in the Fig. 3. The cumulative clearance of SARSCoV-2 was significantly higher in age $\leq 60$ years group than age $>60$ years group $\left(\log \operatorname{Rank} X^{2}=12.15, P<0.001\right)$ (Fig. 3a). The cumulative clearance of SARS-CoV-2 was significantly higher in interferon group compared to non-interferon group ( $\log \operatorname{Rank} X^{2}=8.272, P=0.004$ ) (Fig. 3b).

As a sensitivity analysis, we further analyzed the associated factors of SARS-CoV-2 clearance using date of hospital admission as the start of follow-up. The results revealed that atomized inhalation of interferon $\alpha-2 b(H R, 1.610,95 \%$ CI 1.210-2.142, $P=0.001)$ could promote the clearance of SARS-CoV-2 compared with patients not treated with atomized inhalation of interferon $\alpha-2 b$, while age $>60$ years (HR, 0.630, 95\% CI $0.445-0.890, P=0.009)$ was associated with a prolonged time to SARS-CoV-2 clearance compared with 
Table 4 Cox regression analysis of factors for the clearance of SARS-CoV-2 using date of initial symptoms as the start of follow-up

\begin{tabular}{|c|c|c|c|c|}
\hline \multirow[t]{2}{*}{ Variables } & \multicolumn{2}{|l|}{ Univariate } & \multirow{2}{*}{$\begin{array}{l}\text { Multivariate } \\
\text { HR }(95 \% \mathrm{Cl})\end{array}$} & \multirow[b]{2}{*}{$P$ value } \\
\hline & HR $(95 \% \mathrm{Cl})$ & $P$ value & & \\
\hline \multicolumn{5}{|l|}{ Age (year) } \\
\hline$\leq 60$ & Reference & & & \\
\hline$>60$ & $0.610(0.450,0.826)$ & 0.001 & $0.643(0.454,0.911)$ & 0.013 \\
\hline \multicolumn{5}{|l|}{ Sex } \\
\hline Female & Reference & & & \\
\hline Male & $0.992(0.798,1.233)$ & 0.942 & $0.916(0.721,1.165)$ & 0.475 \\
\hline \multicolumn{5}{|l|}{$\mathrm{BMI}\left(\mathrm{kg} / \mathrm{m}^{2}\right)$} \\
\hline$<28$ & Reference & & & \\
\hline$\geq 28$ & $1.049(0.751,1.466)$ & 0.778 & $1.155(0.817,1.631)$ & 0.415 \\
\hline \multicolumn{5}{|l|}{ Hypertension } \\
\hline No & Reference & & & \\
\hline Yes & $0.743(0.549,1.006)$ & 0.055 & $0.905(0.646,1.269)$ & 0.564 \\
\hline \multicolumn{5}{|l|}{ Diabetes } \\
\hline No & Reference & & & \\
\hline Yes & $0.764(0.515,1.134)$ & 0.181 & $0.869(0.561,1.346)$ & 0.528 \\
\hline \multicolumn{5}{|l|}{ Lymphocytes } \\
\hline No decreased & Reference & & & \\
\hline Decreased & $0.885(0.698,1.123)$ & 0.316 & & \\
\hline \multicolumn{5}{|l|}{$\mathrm{ALT}(\mathrm{U} / \mathrm{L})$} \\
\hline$\leq 40$ & Reference & & & \\
\hline$>40$ & $1.057(0.801,1.396)$ & 0.696 & & \\
\hline \multicolumn{5}{|l|}{ LDH (U/L) } \\
\hline$\leq 250$ & Reference & & & \\
\hline$>250$ & $0.870(0.697,1.086)$ & 0.217 & $0.935(0.722,1.210)$ & 0.607 \\
\hline \multicolumn{5}{|l|}{$\mathrm{ALB}(\mathrm{g} / \mathrm{L})$} \\
\hline$>35$ & Reference & & & \\
\hline$\leq 35$ & $0.774(0.557,1.075)$ & 0.126 & & \\
\hline \multicolumn{5}{|l|}{ D-dimer (mg/L) } \\
\hline$\leq 0.5$ & Reference & & & \\
\hline$>0.5$ & $0.857(0.625,1.176)$ & 0.340 & & \\
\hline \multicolumn{5}{|c|}{ Atomized inhalation of interferon $a-2 b$} \\
\hline No & Reference & & & \\
\hline Yes & $1.355(1.088,1.688)$ & 0.007 & $1.357(1.050,1.755)$ & 0.020 \\
\hline \multicolumn{5}{|c|}{ Lopinavir/ritonavir treatment } \\
\hline No & Reference & & & \\
\hline Yes & $0.993(0.771,1.279)$ & 0.957 & & \\
\hline
\end{tabular}

$P C R$ polymerase chain reaction, $A L T$ alanine transaminase, $L D H$ lactate dehydrogenase, $A L B$ albumin

patients $\leq 60$ years (Additional file 1: Table S1). Accumulative clearance of SARS-CoV-2 was presented in the Additional file 1: Fig. S1. The cumulative clearance of SARS-CoV-2 was significantly higher in age $\leq 60$ years group than age $>60$ years group $\left(\log \operatorname{Rank} \chi^{2}=18.89\right.$, $P<0.001$ ) (Additional file 1: Fig. S1A). The cumulative clearance of SARS-CoV-2 was significantly higher in interferon group compared to non-interferon group $\left(\log \operatorname{Rank} \chi^{2}=12.23, P<0.001\right)$ (Fig. S1B).
The comparisons of clinical characteristics between interferon group and non-interferon group were performed in the Additional file 1: Table S2. The results showed that more patients developed respiratory failure ( $12.9 \%$ vs. $3.9 \%, P=0.003)$ and were admitted to ICU $(10.2 \%$ vs. $2.8 \%, P=0.005)$ in non-interferon group than interferon group. However, the demographic characteristics, laboratory and radiology findings were comparable between these two groups. 

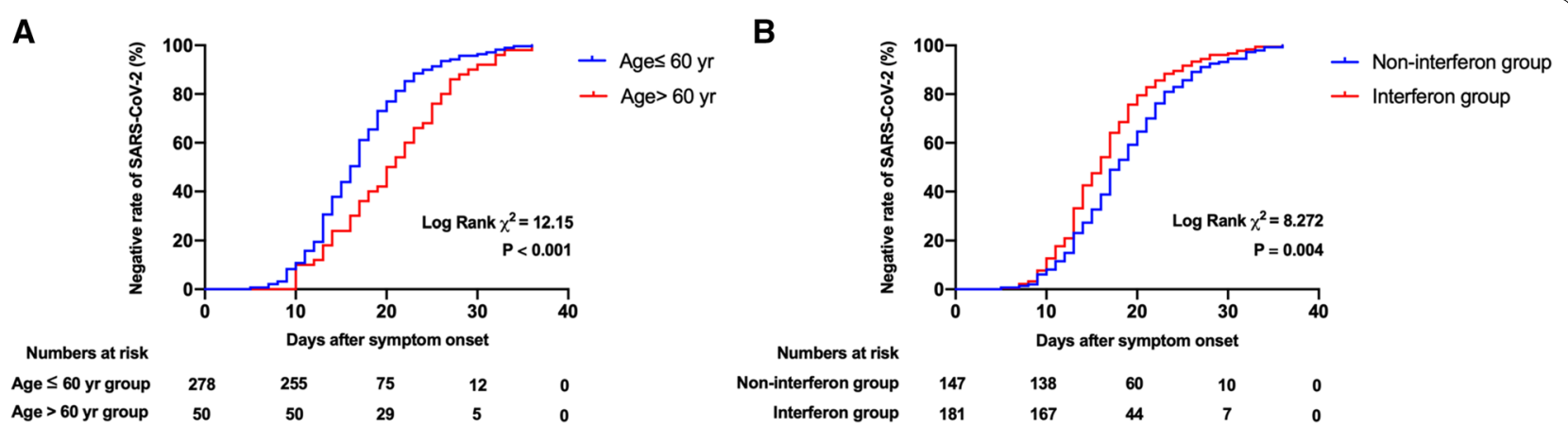

Fig. 3 The cumulative clearance of SARS-CoV-2 in different age and treatment groups using date of initial symptoms as the start of follow-up

\section{Discussion}

Our study described the clinical characteristics of 328 laboratory-confirmed cases of COVID-19 in 10 cities of Jiangsu province, China. Consistent with previous studies, fever and cough are the most common symptoms of COVID-19 in our study $[4,5]$. Over $90 \%$ of the patients had abnormal chest CT results and ground-glass opacities are the most common findings in chest CT images. As of March 10, 2020, all patients were discharged, and no patient died in our study. The clinical outcomes of patients in our study are better than early cases reported in Wuhan with the fatality rate of $11.0-23.8 \%[7,16]$. In the summary of a report of 72, 314 Cases from the Chinese Center for Disease Control and Prevention, the overall case-fatality rate of COVID-19 was 2.3\% [3]. Yang et al. reported 52 critically ill adult patients with SARS-CoV-2 pneumonia admitted to the ICU of Wuhan and the 28-day mortality rate is as high as $61.5 \%$ [17]. However, no deaths were reported among mild and severe cases [3]. In our study, only $13(5.4 \%)$ patients were admitted to the ICU while all patients were recovered after treatment. The possible interpretation of the favorable outcomes in our study may be that the patients were younger and had less comorbidities than previous reports. Previous studies have demonstrated that elder and with more comorbidities were risk factors of admission to ICU and deceased $[6,18]$. These results suggested that age and comorbidity may be risk factors for poor outcome. Another potential explanation of this different outcomes is the lack of medical resources in Wuhan during the outbreak of diseases due to the larger number of patients. However, in Jiangsu, there are enough medical facilities and less patients. Thus, readily available medical care could be easily accessed by and provided to SARSCoV-2 infected patients which may have attributed to the reduced mortality of COVID-19 patients in Jiangsu.

The assessment of virus replication duration is important for evaluating the risk of transmission and guiding the isolation of patients. However, the duration of SARSCoV-2 infection has not been well characterized. Zhou et al. reported that the detectable SARS-CoV-2 RNA persisted for a median of 20 days in survived COVID-19 patients and it was sustained in non-survivors of COVID19 until death in Wuhan [7]. Our study found that the detectable SARS-CoV-2 RNA persisted for a median of 17 days which is shorter than patients reported in Wuhan (median 20.0 days).

The factors of viral clearance were analyzed in our study. We found that older age was associated with delayed clearance of SARS-CoV-2. Previous studies also found that older age was associated with prolonged SARS-CoV-2 RNA shedding [19, 20]. This may be explained by alteration of immune system related to age and ageing that could manifest as reduced immunity and defense to infection [21]. Therefore, more attention should be paid to older patients. In addition, atomized inhalation of interferon $\alpha-2 b$ could improve viral clearance. Currently, no specific treatment is available for COVID-19. Although most of the patients received antiviral therapy including atomized inhalation of interferon $\alpha-2 b$, lopinavir/ritonavir and arbidol. The efficacy of these antiviral agents for COVID-19 is not yet clear. In the previous study, lopinavir/ritonavir treatment was not observed to shorten the clearance of SARS-CoV-2 [7]. Our study also confirmed that lopinavir/ritonavir treatment was not associated with the clearance of SARS-CoV-2. A randomized, controlled, open-label trial enrolled 99 COVID-19 patients with lopinavir-ritonavir group, and 100 COVID-19 patients with standard-care group has also found that the proportion of patients with detectable SARS-CoV-2 RNA were comparable at various time points. In our study, the arbidol treatment was not found to be associated with the clearance of SARS-CoV-2. Another study also reported that arbidol could not accelerate the SARSCoV-2 clearance [22]. However, the results should be 
confirmed in larger prospective randomized studies. Atomized inhalation of interferon $\alpha-2 b$ is recommended for the treatment of COVID-19 in the guidelines for COVID-19 by the Chinese National Health Commission [23]. Previous study also reported the effectiveness of interferon $\alpha-2 b$ in middle east respiratory syndrome coronavirus infection [24, 25]. In COVID-19 patients, previous study also reported that inhaled interferon accelerated the clearance of SARSCoV-2 [26, 27]. A retrospective study which enrolled 77 COVID-19 patients revealed that treatment with atomized inhalation of interferon $\alpha-2 b$ significantly reduced the duration of detectable virus in COVID19 patients [27]. However, the data in our study was from the beginning of the COVID-19 outbreak, and SARS-CoV-2 variant was rare at that time. Recently, numerous studies reported SARS-CoV-2 variant was common in new cases of COVID-19 [28-31]. These studies demonstrated that mutated SARS-CoV-2 spreads faster and may be more virulent $[28,29]$. Therefore, the improved viral clearance by atomized inhalation of interferon $\alpha-2 b$ as demonstrated in our study need to be confirmed in SARS-CoV-2 variant cohort.

This study has several limitations. First, only 328 patients with confirmed COVID-19 were included in the studies from 10 cities of Jiangsu province. We could not include all COVID-19 patients. However, the patients were included from 10 cities of Jiangsu province $(54.2 \%)$. Thus, our study is representative of hospitalized cases in Jiangsu province. Secondly, there was potential ascertainment bias due to the fact that the interval between serial specimens for SARS-CoV-2 testing was not scheduled or standardized in our study. This is a retrospective study and no specific study protocol had been designed for scheduled specimen collection for SARS-CoV-2 testing, which were collected according to clinical condition as assessed by clinicians for clinical management during hospitalization. We cannot exclude the possibility that certain groups of patients would have had specimens collected at more frequent intervals that would have increased chance of having specimens with positive results. Moreover, data on clearance of SARS-CoV-2 might be influenced by the time point in the patients' infection given the time of symptoms onset was reported by patients and recall bias might exist. Furthermore, the retrospective study design does not allow for causation to be determined with regards to the factors associated with the clearance of SARS-CoV-2 in COVID-19 patients. Finally, the association between clearance of SARS-CoV-2 and fatal outcome of patients could not be analyzed.

\section{Conclusions}

The median duration of viral clearance after COVID19 symptom onset was 17.0 days. Age $>60$ years was associated with delayed clearance of while inhalation of atomized interferon alpha-2b hastened clearance of SARS-CoV-2. However, whether atomized inhalation of interferon $\alpha-2 b$ may improve the clinical outcomes and reduce the death of COVID-19 deserve further investigation.

\begin{abstract}
Abbreviations
COVID-19: Coronavirus disease 2019; SARS-CoV-2: Severe acute respiratory syndrome coronavirus 2; RT-PCR: Real-time reverse transcription polymerase chain reaction; ARDS: Acute respiratory distress syndrome; IQR: Interquartile range; BMI: Body mass index.
\end{abstract}

\section{Supplementary Information}

The online version contains supplementary material available at https://doi. org/10.1186/s12985-021-01615-y.

Additional file 1 Fig. S1 The cumulative clearance of SARS-CoV-2 in different age and treatment groups using date of hospital admission as the start of follow up. Table S1. Cox regression analysis of factors for the clearance of SARS-CoV-2 using date of hospital admission as the start of follow-up. Table S2. Clinical characteristics of COVID-19 patients treated with interferon and without interferon.

\section{Acknowledgements}

Not applicable.

\section{Authors' contributions}

Concept and design: Chao Wu, Chuanwu Zhu and Longgen Liu. Drafting of the manuscript: Rui Huang, Jian Wang, Li Zhu, Yuxin Chen; Critical revision of the manuscript for important intellectual content: Yuxin Chen, Chuanwu Zhu, Longgen Liu and Xuebing Yan. Statistical analysis: Jian Wang, Rui Huang, Biyun Xu. Administrative, technical, or material support: Longgen Liu, Haiyan Zhao. Supervision: Chuanwu Zhu, Chao Wu and Longgen Liu. Acquisition, analysis, or interpretation of data: Rui Huang, Jian Wang, Songping Huang, Jie Wei, Juan Xia, Jiacheng Liu, Xiaomin Yan, Xiang-an Zhao, Fang Ming, Li Zhu, Yinling Wang, Biao Zhang, Leyang Xue, Shuqin Hong, Tianmin Xu, Kang Chen, Fang Ji, Chunyang Li, Xuebing Yan, Yun Zhao, Yang Li, Juan Cheng, Lei Zou, Dawen Sang, Huaping Shao, Haiyan Zhao, Xinying Guan and Xiaobing Chen. All authors read and approved the final manuscript.

\section{Funding}

This study was supported by the Fundamental Research Funds for the Central Universities (No. 14380459), Yangzhou Key R\&D Program (Social Development) (No. YZ2020101), China Postdoctoral Science Foundation for COVID-19 (No. 2020T130049ZX).

\section{Availability of data and materials}

The data that support the findings of this study are available from the corresponding author upon reasonable request.

\section{Declarations}

Ethics approval and consent to participate

The study was approved by the institutional ethics board of these hospitals, with a waiver of informed consent.

Consent to publication

All authors reviewed and approved the final version. 


\section{Competing interests}

The authors have declared that no conflicts of interest exist.

\section{Author details}

'Department of Infectious Diseases, Nanjing Drum Tower Hospital, The Affiliated Hospital of Nanjing University Medical School, Nanjing, China. ${ }^{2}$ Department of Infectious Diseases, The Affiliated Infectious Diseases Hospital of Soochow University, Suzhou, China. ${ }^{3}$ Department of Critical Medicine, Huai'an No. 4 People's Hospital, Huai'an, China. ${ }^{4}$ Department of Infectious Diseases, Affiliated Hospital of Xuzhou Medical University, Xuzhou, China. ${ }^{5}$ Department of Infectious Diseases, Nantong Third People's Hospital, Nantong University, Nantong, China. ${ }^{6}$ Department of Quality Control Office, Huai'an No. 4 People's Hospital, Huai'an, China. ${ }^{7}$ Department of Infectious Diseases, The Third People's Hospital of Changzhou, Changzhou, China. ${ }^{8}$ Department of Infectious Diseases, The Third People's Hospital of Yangzhou, Yangzhou, China. ${ }^{9}$ Department of Infectious Diseases, Taizhou People's Hospital, Taizhou, China. ${ }^{10}$ Department of Infectious Diseases, Yancheng Second People's Hospital, Yancheng, China. ${ }^{11}$ Department of Infectious Diseases, The People's Hospital of Suqian, Suqian, China. ${ }^{12}$ Nursing Department, Huai'an No. 4 People's Hospital, Huai'an, China. ${ }^{13}$ Department of Tuberculosis, The Third People's Hospital of Changzhou, Changzhou, China. ${ }^{14}$ Department of Gastroenterology, Northern Jiangsu People's Hospital, Clinical Medical College of Yangzhou University, Yangzhou, China. ${ }^{15}$ Department of Neurology, The Affiliated Hospital of Kangda College of Nanjing Medical University, The First People's Hospital of Lianyungang, Lianyungang, China. ${ }^{16}$ Department of Emergency, The Affiliated Hospital of Kangda College of Nanjing Medical University, The First People's Hospital of Lianyungang, Lianyungang, China. ${ }^{17}$ Department of Biostatistics, Nanjing Drum Tower Hospital, The Affiliated Hospital of Nanjing University Medical School, Nanjing, China. ${ }^{18}$ Department of Laboratory Medicine, Nanjing Drum Tower Hospital, The Affiliated Hospital of Nanjing University Medical School, Nanjing, China. ${ }^{19}$ Department of Infectious Diseases, Nanjing Drum Tower Hospital Clinical College of Nanjing Medical University, Nanjing, China. ${ }^{20}$ Department of Infectious Diseases, Nanjing Drum Tower Hospital Clinical College of Traditional Chinese and Western Medicine, Nanjing University of Chinese Medicine, Nanjing, China.

Received: 18 February 2021 Accepted: 2 July 2021

Published online: 14 July 2021

\section{References}

1. Zhu N, Zhang D, Wang W, Li X, Yang B, Song J, et al. A Novel coronavirus from patients with pneumonia in China, 2019. N Engl J Med. 2020;382:727-33.

2. WHO Coronavirus Disease (COVID-19) Dashboard. https://covid19.who. int/. (Accessed May 14, 2021).

3. Wu Z, McGoogan JM. Characteristics of and important lessons from the coronavirus disease 2019 (COVID-19) outbreak in china: summary of a report of 72314 cases from the Chinese center for disease control and prevention. JAMA. 2020;323:1239-42.

4. Guan WJ, Ni ZY, Hu Y, Liang WH, Ou CQ, He JX, et al. Clinical characteristics of coronavirus disease 2019 in China. N Engl J Med. 2020;382:1708-20.

5. Huang C, Wang Y, Li X, Ren L, Zhao J, Hu Y, et al. Clinical features of patients infected with 2019 novel coronavirus in Wuhan. China Lancet. 2020;395:497-506.

6. Wang D, Hu B, Hu C, Zhu F, Liu X, Zhang J, et al. Clinical characteristics of 138 hospitalized patients with 2019 novel coronavirus-infected pneumonia in Wuhan. China JAMA. 2020;323:1061-9.

7. Zhou F, Yu T, Du R, Fan G, Liu Y, Liu Z, et al. Clinical Course and risk factors for mortality of adult inpatients with COVID-19 in Wuhan, China: a Retrospective Cohort Study. Lancet. 2020;395:1054-62.

8. Xu X, Wu X, Jiang X, Xu KJ, Ying LJ, Ma CL, et al. Clinical findings in a group of patients infected with the 2019 novel coronavirus (SARS-Cov-2) outside of Wuhan, China: retrospective case series. BMJ. 2020;368:m606.

9. Tian S, Hu N, Lou J, Chen K, Kang X, Xiang Z, et al. Characteristics of COVID-19 infection in Beijing. J Infect. 2020;80:401-6.

10. Yang W, Cao Q, Qin L, Wang X, Cheng Z, Pan A, et al. Clinical characteristics and imaging manifestations of the 2019 novel coronavirus disease (COVID-19): a multi-center study in Wenzhou City, Zhejiang. China J Infect. 2020;80:388-93.
11. World Health Organization. Clinical management of severe acute respiratory infection when novel coronavirus ( $\mathrm{nCoV}$ ) infection is suspected: interim guidance. Published January 28, 2020. Accessed January 31, 2020 https://www.who.int/publications-detail/clinical-managementof-severeacute-respiratory-infection-when-novelcoronavirus-(ncov)-infection-issuspected.

12. ARDS Definition Task Force, Ranieri VM, Thompson BT, Ferguson ND, Cald-

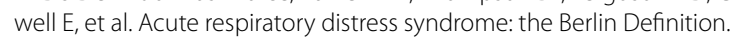
JAMA. 2012:307:2526-33.

13. National Health Commission. Guidelines for the Diagnosis and Treatment of coronavirus disease 2019 (COVID-19) by the National Health Commission (Trial Version 5). (http://www.nhc.gov.cn/yzygj/s7653p/202002/ 3b09b894ac9b4204a79db5b8912d4440.shtml) (Accessed Feb 16, 2020).

14. World Health Organization. Laboratory diagnostics for novel coronavirus 2020. (https://www.who.int/health-topics/coronavirus/laboratory-diagn ostics-for-novel-coronavirus) (Accessed Feb 6, 2020).

15. Jiangsu Provincial Center for Disease Control and Prevention. Novel Coronavirus (COVID-19) Situation. http://www.jshealth.com/xxgk/yqdt/ 202003/t20200301_68110.html. (Accessed March 01, 2020).

16. Chen N, Zhou M, Dong X, Qu J, Gong F, Han Y, et al. Epidemiological and clinical characteristics of 99 cases of 2019 novel coronavirus pneumonia in Wuhan, China: a descriptive study. Lancet. 2020;395:507-13.

17. Yang $X, Y u Y, X u$ J, Shu H, Xia J, Liu H, et al. Clinical course and outcomes of critically ill patients with SARS-CoV-2 pneumonia in Wuhan, China: a single-centered, retrospective, observational study. Lancet Respir Med. 2020:8:475-81.

18. Chen T, Wu D, Chen H, Yan W, Yang D, Chen G, et al. Clinical characteristics of 113 deceased patients with coronavirus disease 2019: retrospective study. BMJ. 2020;368:1091.

19. Chen X, Zhu B, Hong W, Zeng J, He X, Chen J, et al. Associations of clinical characteristics and treatment regimens with the duration of viral RNA shedding in patients with COVID-19. Int J Infect Dis. 2020;98:252-60.

20. Huang R, Zhu C, Wang J, Xue L, Li C, Yan X et al (2020) Corticosteroid therapy is associated with the delay of SARS-CoV-2 clearance in COVID-19 patients. Eur J Pharmacol 889:173556.

21. Sadighi Akha AA. Aging and the immune system: an overview. J Immunol Methods. 2018;463:21-6.

22. Li Y, Xie Z, Lin W, Cai W, Wen C, Guan Y, et al. Efficacy and safety of lopinavir/ritonavir or arbidol in adult patients with mild/moderate COVID-19: an exploratory randomized controlled trial. Medicine. 2020;1:105-13.

23. Du B, Qiu HB, Zhan X, Wang YS, Kang HYJ, Li XY, et al. Pharmacotherapeutics for the new coronavirus pneumonia. Chin J Tuberc Respir Dis. 2020;43:173-6.

24. Khalid M, Al Rabiah F, Khan B, Al Mobeireek A, Butt TS, Al ME. Ribavirin and interferon-a2b as primary and preventive treatment for middle east respiratory syndrome coronavirus: a preliminary report of two cases. Antivir Ther. 2015;20:87-91.

25. Singh SK. Middle east respiratory syndrome virus pathogenesis. Semin Respir Crit Care Med. 2016;37:572-7.

26. Andreakos E, Tsiodras S. COVID-19: lambda interferon against viral load and hyperinflammation. EMBO Mol Med. 2020;12:e12465.

27. Zhou Q, Chen V, Shannon CP, Wei X-S, Xiang X, Wang X, et al. Interferona2b treatment for COVID-19. Front Immunol. 2020;11:1061.

28. Garcia-Beltran WF, Lam EC, St Denis K, Nitido AD, Garcia ZH, Hauser BM, et al. Multiple SARS-CoV-2 variants escape neutralization by vaccineinduced humoral immunity. Cell. 2021;184:2372-83.e9.

29. Davies NG, Abbott S, Barnard RC, Jarvis Cl, Kucharski AJ, Munday JD, et al. Estimated transmissibility and impact of SARS-CoV-2 lineage B.1.1.7 in England. Science. 2021;372:eabg3055.

30. Buenestado-Serrano S, Recio R, Sola Campoy PJ, Catalán P, Folgueira MD, Villa J, et al. First confirmation of importation and transmission in Spain of the newly identified SARS-CoV-2 B.1.1.7 variant. Enferm Infecc Microbiol Clin. 2021. [Epub ahead of print].

31. Xie X, Liu Y, Liu J, Zhang X, Zou J, Fontes-Garfias CR, et al. Neutralization of SARS-CoV-2 spike 69/70 deletion, E484K and N501Y variants by BNT162b2 vaccine-elicited sera. Nat Med. 2021;27:620-1.

\section{Publisher's Note}

Springer Nature remains neutral with regard to jurisdictional claims in published maps and institutional affiliations. 